\title{
Legionella nagasakiensis sp. nov., isolated from water samples and from a patient with pneumonia
}

Correspondence
Genyan Yang
gyang@cdc.gov
Genyan Yang, ${ }^{1,2}$ Robert F. Benson, ${ }^{2}$ Rodney M. Ratcliff, ${ }^{3}$ Ellen W. Brown, ${ }^{2}$ Arnold G. Steigerwalt, ${ }^{4}$ W. Lanier Thacker, ${ }^{2}$ Maryam I. Daneshvar, ${ }^{5}$ Roger E. Morey, ${ }^{4}$ Atsushi Saito ${ }^{6} \dagger$ and Barry S. Fields ${ }^{2}$

${ }^{1}$ Division of Blood Disorders, National Center on Birth Defects and Developmental Disabilities, Centers for Disease Control and Prevention, Atlanta, GA, USA

${ }^{2}$ Division of Bacterial Diseases, National Center for Immunization and Respiratory Diseases, Centers for Disease Control and Prevention, Atlanta, GA, USA

${ }^{3}$ Microbiology and Infectious Diseases, Institute of Medical and Veterinary Science, SA Pathology, and School of Molecular \& Biomedical Science, University of Adelaide, Adelaide, Australia

${ }^{4}$ Division of Foodborne, Bacterial, and Mycotic Diseases, National Center for Zoonotic, Vector-Borne, and Enteric Diseases, Centers for Disease Control and Prevention, Atlanta, GA, USA

${ }^{5}$ Laboratory Science, Policy, and Practice Program Office; Office of Surveillance, Epidemiology and Laboratory Services, Centers for Disease Control and Prevention, Atlanta, GA, USA

${ }^{6}$ Department of Internal Medicine, Nagasaki University Hospital, Nagasaki, Japan

A novel Legionella species was identified based on analysis of 16S rRNA and mip (macrophage infectivity potentiator) gene sequences, cellular fatty acids, isoprenoid quinones, biochemical reactions, antigens and quantitative DNA-DNA hybridization. Strain CDC-1796-JAP-E ${ }^{\top}$ was isolated from well water at the Nagasaki Municipal Medical Center, Japan. Two strains, CDC-3041-AUS-E and CDC-3558-AUS-E, were isolated from water samples during an outbreak of legionellosis in South Australia. Strain CDC-5427-OH-H was isolated from a 66-year-old female patient diagnosed with Legionnaires' disease in the US. Cells from these four strains were Gram-negative, non-fluorescent, rod-shaped, and positive for alkaline phosphatase, esterase, leucine arylamidase, catalase, gelatinase, $\beta$-lactamase and tyrosine browning assay. Phylogenetic analysis of $16 \mathrm{~S}$ rRNA and mip genes revealed that the four strains formed a distinct cluster within the genus Legionella. The bacteria contained branched-chain fatty acids and quinones that are typical of members of the genus Legionella. Slide agglutination tests demonstrated no cross-reaction with 52 previously described members of the Legionellaceae. DNA-DNA hybridization studies indicated that DNAs from the four strains were highly related (78-84 \%) but they showed $29 \%$ relatedness to Legionella oakridgensis ATCC $33761^{\top}$ and less than $10 \%$ to strains of other Legionella species tested. These characterizations suggest that the isolates represent a novel species, for which the name Legionella nagasakiensis sp. nov. is proposed; the type strain is CDC-1796-JAP-E ${ }^{\top}\left(=\right.$ ATCC BAA-1557 $\left.^{\top}=\mathrm{JCM}^{15315^{\top}}\right)$.
†Present address: San-Remo Rehabilitation Hospital, Sasebo, Nagasaki, Japan.

Abbreviations: CFA, cellular fatty acids; LLOs, Legionella-like organisms; mip, macrophage infectivity potentiator.

The GenBank/EMBL/DDBJ accession numbers for the 16S rRNA gene sequences of strains CDC-3558-AUS-E, CDC-3041-AUS-E, CDC1796-JAP-E ${ }^{\top}$ and CDC-5427-OH-H are FJ236837, FJ236838, EU701006 and EU701007, respectively. The GenBank/EMBL/DDBJ accession numbers for the mip gene sequences of strains CDC-3041AUS-E, CDC-1796-JAP-E', CDC-5427-OH-H and CDC-3558-AUS-E are FJ236839, FJ236840, FJ236841 and FJ236842, respectively.

A supplementary figure and two supplementary tables are available with the online version of this paper.
The genus Legionella belongs to the family Legionellaceae. Fifty-three Legionella species containing 73 serogroups have been described (Lück et al., 2010). More than 40 additional isolates have been identified as Legionella-like organisms (LLOs) in the Centers for Disease Control and Prevention strain collection (unpublished results). These LLOs resemble members of the Legionellaceae morphologically and require L-cysteine for growth. Legionellaceae are ubiquitous in soils, and in natural and man-made aquatic environments (Fields et al., 2002). At least 24 species of Legionella have been reported as being pathogenic in humans (Newton et al., 2010). The pathogenic species 
cause legionellosis, a significant respiratory disease that classically takes two distinct clinical forms: Legionnaires' disease, a severe multisystem disease involving pneumonia; or Pontiac Fever, a milder flu-like illness (Fields et al., 2002). In this paper, the growth, macrophage infectivity potentiator (mip) and 16S rRNA gene sequences, physiological characteristics, cellular fatty acid (CFA) composition, serological characteristics, DNA relatedness data of a novel Legionella species are described. Strains of this novel species, named Legionella nagasakiensis sp. nov., were isolated from well water in Japan, cooling tower water in Australia and a patient with pneumonia in the United States.

Strain CDC-1796-JAP-E ${ }^{\mathrm{T}}$ was isolated from well water in Nagasaki, Japan, using the following method. A $500 \mathrm{ml}$ sample was collected in sterile bottles and centrifuged at 10000 r.p.m. for $20 \mathrm{~min}$. The supernatant was decanted and $5.0 \mathrm{ml}$ sediment was removed, acid-treated and plated on selective BCYE (buffered charcoal-yeast extract) medium supplemented with PAV (polymyxin B, anisomycin and vancomycin) (Bopp et al., 1981). Plates were incubated at $35{ }^{\circ} \mathrm{C}$ and examined for growth of Legionella colonies. Colonies resembling Legionella were subcultured on BCYE agar. Formalin-fixed cells were stained with direct immunofluorescence conjugates for Legionella pneumophila serogroups 1-6, Legionella micdadei, Legionella dumoffii, Legionella bozemanae serogroup 1, Legionella gormanii and Legionella longbeachae serogroup 1. Strain CDC-1796-JAP$\mathrm{E}^{\mathrm{T}}\left(=82-059^{\mathrm{T}}\right)$ failed to react with these antisera and was forwarded to CDC for further studies. Strains IMVS 86 (=CDC-3041-AUS-E) and IMVS 934 (=CDC-3558-AUSE) were received from the Institute of Medical and Veterinary Science, Adelaide, South Australia. These strains were previously designated 'species B' (Wilkinson et al., 1990). Strain CDC-5427-OH-H was received from Ohio State University Hospital East, Columbus, Ohio, USA, and had been isolated from a bronchial alveolar lavage specimen obtained from a 66-year-old female patient diagnosed with Legionnaires' disease.

All strains were streaked for single colony isolation on BCYE agar. The ACES buffer was omitted for determination of autofluorescence and L-cysteine was omitted for the determination of cysteine requirement. Physiological tests for flagella, autofluorescence and the enzymes listed in Table 1 were performed using API ZYM (bioMérieux) according to the manufacturer's instructions or as described previously (Vesey et al., 1988). All isolates were tested with antisera to previously characterized Legionella species available to us $(n=52)$ and serogroups $(n=74)$ (Benson et al., 1996; Dennis et al., 1993; Thacker et al., 1992; Verma et al., 1992). Antisera to the novel isolates (CDC-1796-JAP-E ${ }^{\mathrm{T}}$, CDC-3041-AUS-E and CDC-3558AUS-E) were prepared and tested as described previously (Thacker et al., 1985).

DNA for sequence determination was extracted using a QIAamp DNA Mini kit (Qiagen). 16S rRNA gene was amplified using the Expand high-fidelity PCR system (Roche) with primers fD1 and rp2 (Weisburg et al., 1991). The mip gene was amplified as described previously (Ratcliff et al., 1998). Sequencing reactions were performed on at least two separately generated amplification products to minimize the possibility of sequencing errors. Each set of gene target sequences was phylogenetically compared with the published type strains of each species. Additional sequencing of the 16S rRNA genes from some species was performed to remove ambiguities from published sequences. Sequence assembly, alignment and analysis were performed using Kodon (Applied Maths) and phylogenetic analysis (maximum-likelihood) was performed using PAUP 4 (Sinauer Associates). The best fit model GTR + I + G used for the analysis was assessed using Modeltest 3.7 (Posada \& Crandall, 1998) and relationship trees were drawn with FigTree v1.1.2 (A. Rambaut, Institute of Evolutionary Biology, University of Edinburgh, UK).

For CFA and isoprenoid quinone analyses, all strains were suspended in water and diluted to the same concentration (based on optical density readings of 0.9 at $600 \mathrm{~nm}$ ). A bacterial suspension of $100 \mu \mathrm{l}$ was streaked on BCYE agar plates and cultured at $35{ }^{\circ} \mathrm{C}$ with $2.5 \% \mathrm{CO}_{2}$ for $48 \mathrm{~h}$. Cells harvested from the second quadrant were saponified and processed and CFA (as methyl esters) were analysed by capillary GLC as described previously (Lambert \& Moss, 1989). Isoprenoid quinones were extracted from 100$250 \mathrm{mg}$ lyophilized cells and analysed by reverse-phase HPLC (Moss \& Guerrant, 1983). Individual fatty acids and quinones were tentatively identified by retention time comparisons to known standards and were subsequently confirmed by MS (Lambert \& Moss, 1989).

Relative binding ratios were determined using the freesolution hydroxyapatite method performed at $60{ }^{\circ} \mathrm{C}$ (Brenner et al., 1982). In vitro labelling of DNA was performed enzymically by using $\left[{ }^{32} \mathrm{P}\right] \mathrm{dCTP}$ provided in a nick-translation reagent kit (Bethesda Research Laboratories), as directed by the manufacturer.

All four isolates (CDC-1796-JAP-E ${ }^{\mathrm{T}}$, CDC-3558-AUS-E, CDC-3041-AUS-E and CDC-5427-OH-H) required Lcysteine for primary isolation, but they were able to adapt to cysteine-deficient media in serial transfer on BCYE agar. The loss of requirement for L-cysteine has also been observed in Legionella oakridgensis and Legionella jordanis (Orrison et al., 1983). Cells from these strains were Gramnegative rods with single polar flagella. Colonies of these strains did not exhibit autofluorescence when exposed to UV light.

Analysis of the 16S rRNA and mip genes of these strains resulted in a discrete cluster of sequences ( $0.1 \%$ variant) within the genus Legionella. Alignment of the 16S rRNA genes of the isolates produced three sequence variants from two positions, with identity shared between strains CDC3558-AUS-E and CDC-1796-JAP-E ${ }^{\mathrm{T}}$ (Supplementary Fig. S1a, available in IJSEM Online). Alignment of mip genes for the four isolates produced three sequence variants from 
Table 1. Biochemical characteristics of $L$. nagasakiensis CDC-1796-JAP-E ${ }^{\top}$ and its most closely related species and controls

Strains: 1, L. nagasakiensis CDC-1796-JAP-E ${ }^{\mathrm{T}}$; 2, L. oakridgensis ATCC $33761^{\mathrm{T}} ; 3$, L. impletisoli DSM 18493 ${ }^{\mathrm{T}}$; 4, L. yabuuchiae DSM 18492 ${ }^{\mathrm{T}}$; 5, L. pneumophila ATCC $33152^{\mathrm{T}}$. +, Positive; w, weak positive; -, negative; NA, not available. Tests 1-19 were performed using API ZYM (bioMérieux) following the instructions. Tests 20-27 were performed as described previously (Vesey et al., 1988). All negative controls were performed using uninoculated media unless otherwise indicated. All positive controls were performed using the indicated bacterial strains or purifed enzymes.

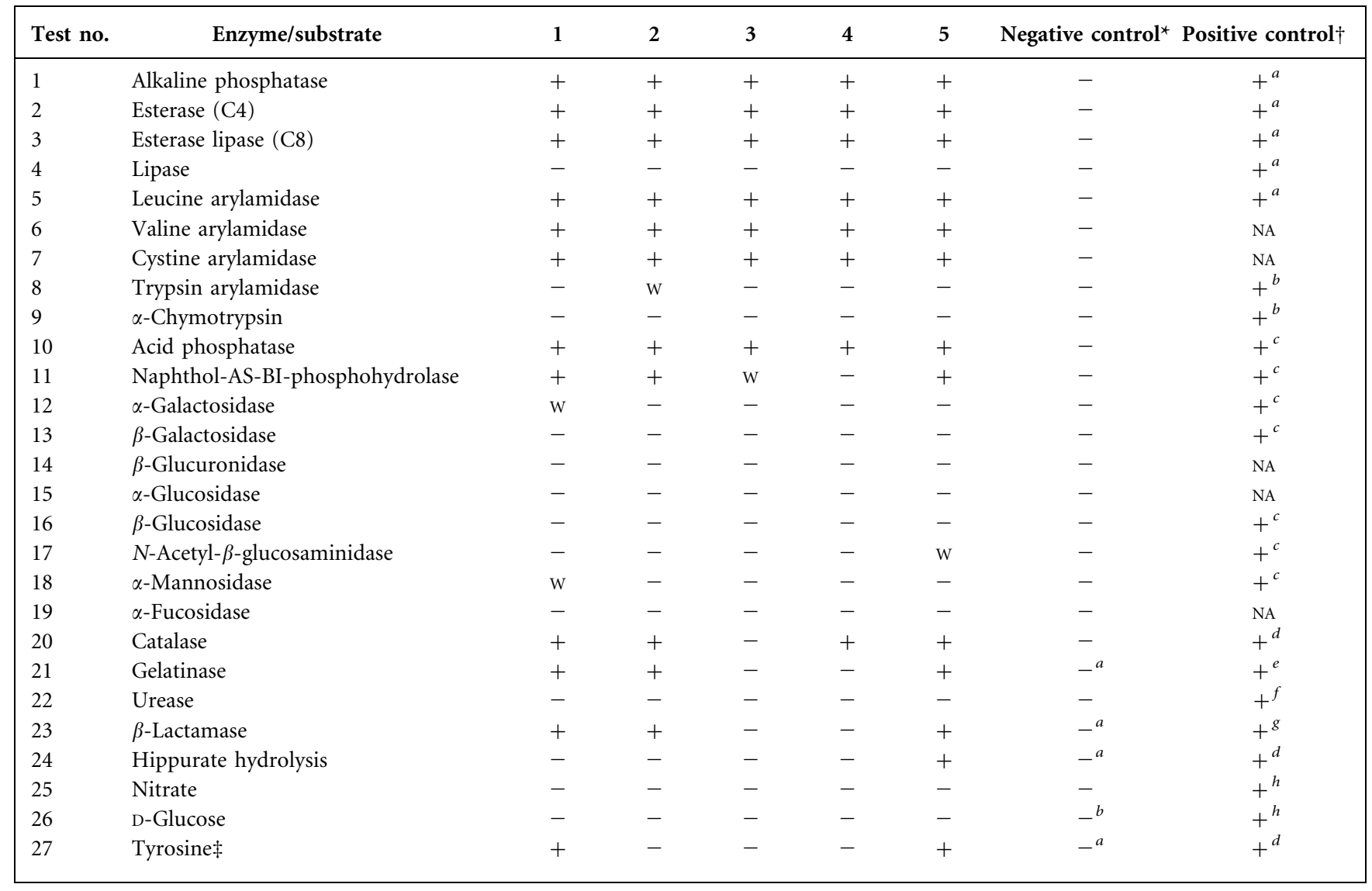

${ }^{\star}$ Controls: $a$, L. micdadei; $b$, L. pneumophila sg1.

$\dagger$ Controls: $a$, Pesudomonas aeruginosa ATCC 27853; $b$, $\alpha$-chymotrypsin (Sigma); $c$, $\beta$-glucosidase (Sigma); $d$, L. pneumophila sg1 strain Knoxville-1; e, L. bozemanae ATCC $33217^{\mathrm{T}}$; f, Providencia rettgeri; g, L. micdadei ATCC $33218^{\mathrm{T}} ;$, E. coli ATCC 25922.

$\$$ Browning with tyrosine was read twice at 3 days and 5 days post-incubation.

three positions. Identity was shared between strains CDC5427-OH-H and CDC-1796-JAP-E ${ }^{\mathrm{T}}$ (Supplementary Fig. S1b, available in IJSEM Online). The isolates showed dissimilarities of $3.5 \%$ and $14.4 \%$ for the $16 \mathrm{~S}$ rRNA and mip gene sequences, respectively, to the type strain of the most closely related species, L. oakridgensis. Such levels of divergence are, for both gene targets, several fold greater than that for existing recognized species. For example, only $1.7 \%$ dissimilarity in $16 \mathrm{~S}$ rRNA gene was observed between L. longbeachae and Legionella sainthelensi, and mip gene sequencing revealed less than $7 \%$ dissimilarity between the tight cluster of species comprising Legionella anisa, $L$. bozemanae, Legionella parisiensis and Legionella tucsonensis (data not shown).

The CFA profiles of saponified cells of the four isolates were essentially identical. Like other Legionella species (Lambert
\& Moss, 1989), they contained significant amounts of branched-chain fatty acids with no or only traces of hydroxyl fatty acids (Supplementary Table S1, available in IJSEM Online). iso- $\mathrm{C}_{16: 0}$ and $\mathrm{C}_{16: 1} \omega 07 c$ were the two major fatty acids. In comparison with the most closely related species, $L$. oakridgensis, the isolates contained twice the amounts of anteiso- $\mathrm{C}_{15: 0}, \mathrm{C}_{15: 1} \omega 7 c, \mathrm{C}_{15: 0}$ and $\mathrm{C}_{17: 0}$ and about half the amounts of iso- $\mathrm{C}_{16: 1}, \mathrm{C}_{17: 0}$ and $\mathrm{C}_{18: 0}$.

Each of the four isolates contained ubiquinone-10 (Q-10) as the major quinone with small amounts of Q-9 and Q-11. This quinone profile is essentially identical to that of $L$. oakridgensis and Legionella wadsworthii that were previously placed together in a separate ubiquinone group (Lambert \& Moss, 1989; Wilkinson et al., 1990).

Antisera prepared against three of the isolates (CDC-3558AUS-E, CDC-3041-AUS-E, CDC-1796-JAP-E ${ }^{\mathrm{T}}$ ) did not 
react to any of the 52 previously described Legionella species or serogroups using the slide agglutination test, including strains of the most closely related species, i.e. $L$. oakridgensis ATCC $33761^{\mathrm{T}}$, Legionella impletisoli DSM $18493^{\mathrm{T}}$ and Legionella yabuuchiae DSM $18492^{\mathrm{T}}$.

Reactions of strain CDC-1796-JAP-E ${ }^{\mathrm{T}}$ in 27 different biochemical tests revealed that the isolate differed from its nearest neighbours and Legionella pneumophila, the type species of the genus Legionella (Table 1). For example, trypsin arylamidase was positive for L. oakridgensis ATCC $33761^{\mathrm{T}}$, but negative for CDC-1796-JAP-E ${ }^{\mathrm{T}}$. On the other hand, CDC-1796-JAP- $\mathrm{E}^{\mathrm{T}}$ was positive for $\alpha$-galactosidase, $\alpha$-mannosidase and tyrosine browning, whereas L. oakridgensis was negative for these reactions. The isolates could be clearly distinguished from L. impletisoli DSM $18493^{\mathrm{T}}$ and L. yabuuchiae DSM $18492^{\mathrm{T}}$ based on tests for naphthol-AS-BI-phosphohydrolase, gelatinase, $\beta$-lactamase and tyrosine browning (Table 1).

DNA relatedness, determined by relative binding ratios at $60{ }^{\circ} \mathrm{C}$, showed that labelled DNA from CDC-1796-JAP-E $\mathrm{E}^{\mathrm{T}}$ was $78.5-83.5 \%$ related to DNA from the other three novel isolates (Supplementary Table S2, available in IJSEM Online). The divergence (\%) in these reactions was $0-0.8 \%$. The levels of relatedness to two strains of $L$. oakridgensis, the most closely related species, were $29 \pm 2.8$ and $35.5 \pm 3.5 \%$ and relatedness was less than $10 \%$ to DNAs of the 32 Legionella species and six LLOs tested (Supplementary Table S2).

This study demonstrated that the environmental and clinical isolates (CDC-1796-JAP-E ${ }^{\mathrm{T}}$, CDC-3558-AUS-E, CDC-3041-AUS-E and CDC-5427-OH-H) represent a novel pathogenic Legionella species, L. nagasakiensis sp. nov. The morphology, biochemical reactions and CFA profiles of these strains are typical of the genus Legionella. They clustered within the family Legionellaceae in both $16 \mathrm{~S}$ rRNA and mip gene phylogenetic trees. Their 16S rRNA genes had $96 \%$ sequence similarity to that of the type strain of L. oakridgensis, but the less conserved mip genes revealed only $85.5 \%$ sequence similarity to the type strain of $L$. oakridgensis. CFA profiles, antiserum agglutination and biochemical reactions of the novel isolates were also different from those of $L$. oakridgensis. Genome-wide DNA-DNA hybridization analysis further proved that the isolates were distinct from $L$. oakridgensis and other Legionella species tested. Although the 16S rRNA and mip genes of all four isolates were nearly identical, it has been reported that genetic analysis that is based on an individual gene is of limited use in serogroup level differentiation of Legionellaceae (Ratcliff et al., 1998). In our studies, we found antisera reacted strongly with their homologous antigens, but presented different patterns for heterologous antigens, suggesting that there are multiple serogroups of $L$. nagasakiensis (data not shown). Indeed, the genome-wide analysis using DNA hybridization demonstrated that the genomes of the L. nagasakiensis strains differed by $17-22 \%$ (Supplementary Table S2).
Description of Legionella nagasakiensis sp. nov.

Legionella nagasakiensis (na.ga.sa.ki.en'sis. N.L. fem adj. nagasakiensis of/from Nagasaki, Japan, where the organism was isolated).

Gram-negative rods, single polar flagella. Grows on BCYE agar and requires L-cysteine for primary isolation. Positive for alkaline phosphatase, esterase, esterase lipase (C8), leucine arylamidase, valine arylamidase, cystine arylamidase, acid phosphatase, naphthol-AS-BI-phosphohydrolase, catalase, gelatinase, $\beta$-lactamase and tyrosine browning. CFA are predominantly branched-chain; major isoprenoid quinone is Q-10. DNA relatedness data and serological reactions indicate that this species is distinct from other members of the Legionellaceae. Associated with human disease as one of the strains was isolated from a pneumonia patient.

The type strain is CDC-1796-JAP-E ${ }^{\mathrm{T}}$ (=ATCC BAA$1557^{\mathrm{T}}=$ JCM $15315^{\mathrm{T}}$ ).

\section{Acknowledgements}

We would like to thank Dr Nick Cianciotto and Dr Cynthia Whitney for their critical review of our manuscript. The findings and conclusions in this report are those of the authors and do not necessarily represent the views of the CDC.

\section{References}

Benson, R. F., Thacker, W. L., Daneshvar, M. I. \& Brenner, D. J. (1996). Legionella waltersii sp. nov. and an unnamed Legionella genomospecies isolated from water in Australia. Int J Syst Bacteriol 46, 631-634.

Bopp, C. A., Sumner, J. W., Morris, G. K. \& Wells, J. G. (1981). Isolation of Legionella spp. from environmental water samples by low-pH treatment and use of a selective medium. J Clin Microbiol 13, 714-719.

Brenner, D. J., McWhorter, A. C., Knutson, J. K. \& Steigerwalt, A. G. (1982). Escherichia vulneris: a new species of Enterobacteriaceae associated with human wounds. J Clin Microbiol 15, 1133-1140.

Dennis, P. J., Brenner, D. J., Thacker, W. L., Wait, R., Vesey, G., Steigerwalt, A. G. \& Benson, R. F. (1993). Five new Legionella species isolated from water. Int J Syst Bacteriol 43, 329-337.

Fields, B. S., Benson, R. F. \& Besser, R. E. (2002). Legionella and Legionnaires' disease: 25 years of investigation. Clin Microbiol Rev 15, 506-526.

Lambert, M. A. \& Moss, C. W. (1989). Cellular fatty acid compositions and isoprenoid quinone contents of 23 Legionella species. J Clin Microbiol 27, 465-473.

Lück, P. C., Jacobs, E., Röske, I., Schröter-Bobsin, U., Dumke, R. \& Gronow, S. (2010). Legionella dresdenensis sp. nov., isolated from river water. Int J Syst Evol Microbiol 60, 2557-2562.

Moss, C. W. \& Guerrant, G. O. (1983). Separation of bacterial ubiquinones by reverse-phase high-pressure liquid chromatography. J Clin Microbiol 18, 15-17.

Newton, H. J., Ang, D. K., van Driel, I. R. \& Hartland, E. L. (2010). Molecular pathogenesis of infections caused by Legionella pneumophila. Clin Microbiol Rev 23, 274-298.

Orrison, L. H., Cherry, W. B., Tyndall, R. L., Fliermans, C. B., Gough, S. B., Lambert, M. A., McDougal, L. K., Bibb, W. F. \& Brenner, D. J. (1983). Legionella oakridgensis: unusual new species isolated from cooling tower water. Appl Environ Microbiol 45, 536-545. 
Posada, D. \& Crandall, K. A. (1998). MODELTEST: testing the model of DNA substitution. Bioinformatics 14, 817-818.

Ratcliff, R. M., Lanser, J. A., Manning, P. A. \& Heuzenroeder, M. W. (1998). Sequence-based classification scheme for the genus Legionella targeting the mip gene. J Clin Microbiol 36, 1560-1567.

Thacker, W. L., Plikaytis, B. B. \& Wilkinson, H. W. (1985). Identification of 22 Legionella species and 33 serogroups with the slide agglutination test. J Clin Microbiol 21, 779-782.

Thacker, W. L., Dyke, J. W., Benson, R. F., Havlichek, D. H., Jr, Robinson-Dunn, B., Stiefel, H., Schneider, W., Moss, C. W., Mayberry, W. R. \& Brenner, D. J. (1992). Legionella lansingensis sp. nov. isolated from a patient with pneumonia and underlying chronic lymphocytic leukemia. J Clin Microbiol 30, 23982401 .
Verma, U. K., Brenner, D. J., Thacker, W. L., Benson, R. F., Vesey, G., Kurtz, J. B., Dennis, P. J., Steigerwalt, A. G., Robinson, J. S. \& Moss, C. W. (1992). Legionella shakespearei sp. nov., isolated from cooling tower water. Int J Syst Bacteriol 42, 404-407.

Vesey, G., Dennis, P. J., Lee, J. V. \& Westy, A. A. (1988). Further development of simple tests to differentiate the legionellas. J Appl Bacteriol 65, 339-345.

Weisburg, W. G., Barns, S. M., Pelletier, D. A. \& Lane, D. J. (1991). $16 \mathrm{~S}$ ribosomal DNA amplification for phylogenetic study. J Bacteriol 173, 697-703.

Wilkinson, I. J., Sangster, N., Ratcliff, R. M., Mugg, P. A., Davos, D. E. \& Lanser, J. A. (1990). Problems associated with identification of Legionella species from the environment and isolation of six possible new species. Appl Environ Microbiol 56, 796-802. 